\title{
Nutritional surveillance in Ireland: theory and practice
}

\author{
BY ALAN KELLY* \\ Department of Statistics, Trinity College, Dublin, Irish Republic
}

A decade has passed since the Food Advisory Committee (FAC) of the Irish Department of Health advocated the establishment of a nutrition policy for Ireland (Food Advisory Committec, 1979). The FAC noted that such a policy should provide for ' . . a national strategy for improving the nutritional status of the population . . . based on the coordinated planning and development of sectoral activities related to the supply, consumption and biological utilization of food'. Further, the FAC considered that ' . . . a national system for the continuous assessment of food and nutrition conditions should be developed on the basis of surveillance indicators and periodic micro-surveys'. At that time a basic nutritional surveillance (NS) system was undergoing development in the Departments of Statistics and Community Health, Trinity College, Dublin, and with the support of the Agricultural Institute. Subsequently, in 1984, the Minister for Health formally established the programme, and to date a number of reports have been produced (Kelly \& Kevany, 1985; Kelly, 1986, 1989a).

The issues which will be considered in the present paper include: the purpose and scope of NS, its relevance to Ireland at this time, and certain practical aspects such as data requirements and analysis. Current constraints on the system are identified and its future needs indicated. These topics receive only a general treatment in the present paper, a more detailed exposition of the rationale and methodology for NS will be found in Mason et al. (1984) and Kelly $(1987,1989 b)$.

\section{THEORY}

What is NS? NS means 'to watch over nutrition in order to make decisions which will lead to improvements in nutrition in populations' (World Health Organization, 1976). Operationally, this may be visualized in terms of the flow of information to planners, its assessment in policy terms, decisions on appropriate sectoral activities, implementation, population impact, followed by a further cycle of data collection and analysis (see Fig. 1). The process is clearly iterative, the purpose being to provide on a continuing basis timely, accurate and relevant information to facilitate detection, control and prevention of nutrition-related public health problems. The focus is entirely pragmatic, data requirements being determined by planning and decision-making needs; in effect, surveillance measures what is necessary for policy guidance, and that alone.

Since the initial specification of a general strategy for NS in the mid-seventies, a number of specialized NS systems have evolved to address particular application areas (Mason et al. 1984; Rothe \& Habicht, 1990). These are:

(1) timely warning and intervention for famine prevention,

(2) programme management and evaluation,

(3) policy and planning in the medium-to-long term.

* Present address: UN Administrative Committee on Coordination/Sub-Committee on Nutrition, c/o WHO, Geneva 27, Switzerland 1211 . 


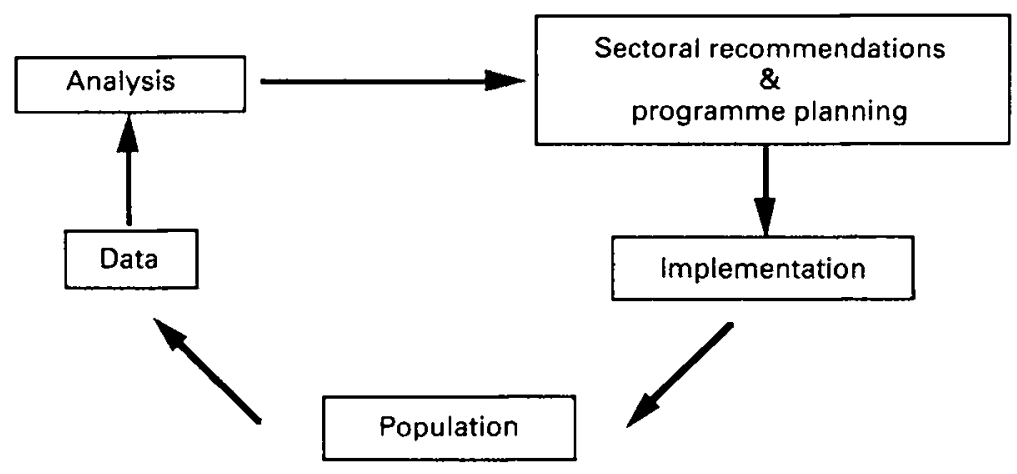

Fig. 1. Information cycle in a nutritional surveillance programme (adopted from ACC/SCN, 1989).

Rothe \& Habicht (1990) report examples of country applications of these forms of NS. (Further extensive activity to develop and deploy additional surveillance capability in upwards of forty developing countries worldwide, is presently being undertaken as a joint FAO/UNICEF/WHO programme, with coordination being provided by the United Nations ACC/SCN (Mason, 1987).) NS for 'timely warning for famine prevention' is clearly irrelevant to the theme of the present paper, however, types 2 and 3 , especially the latter, are now recognized as having a potentially important role to play in a European context (Kelly, 1987; WHO/EURO, 1990).

A role for $N S$ in Ireland. Ireland, in common with most of its European neighbours, has not yet formulated an integrated nutrition policy with fully specified objectives and identified strategies. This poses an immediate difficulty, namely, without a relevant policy foundation and associated targets and goals, what information is required to support which decisions? This does not fit the schema of Fig. 1, as it appears to ignore the right-hand side of the cycle, and it is reasonable to ask under these circumstances: What need for NS? The answer put forward at the beginning of the programme (1979) and offered to the Department of Health in 1984 (and indeed confirmed by subsequent experience), is that by providing basic information on national diet and disease patterns, NS can assist in defining problem areas, motivate and inform the general debate on nutrition issues and provide an impetus to the development of a nutrition policy. This is now acknowledged as an important function for NS, and in addition to the existing three areas a further variety has been recognized, i.e.

(4) NS for nutrition advocacy, both national and international.

Habicht (1988) points out that it is now apparent that most systems previously identified as type 3 are in fact of type 4; the important distinction being that no formal linkages exist between data collection and decision-making. This has certainly been our experience in Ireland. Since its inception, the choice of which data to collect, what analysis to perform and the manner in which findings are reported, has been determined without direction from the official end-user. In the context of the developed world in which the nutrition challenge is by no means elementary (policy options are ill-defined with many possible outcomes and no guarantees of success) this apparent lack of direction from on-high is, up to a point, not critical. When in doubt, hasten slowly! 


\section{THEORY APPLIED}

Information needs. Thus, given the situation in Ireland (or indeed in many other developed and rapidly developing countries) what specifically can a surveillance system contribute? Ideally, a surveillance system needs to:

1. Identify and capture appropriate data,

2. Provide for proper analysis and interpretation,

3. Extract and synthesize useful information,

4. Report findings in a compelling manner.

First we must consider what is meant by 'appropriate' data, which in turn depends on what information is required and how it will be used (information = data processed into a form valuable to the user in current or prospective actions or decisions). It was already noted that no previous stipulation for specific information to either address certain policy questions or monitor current programmes existed. Essentially, the approach taken was to consider the stages through which planning progresses: (1) problem recognition, (2) problem formulation, (3) choice of tactics, (4) implementation, (5) feedback and evaluation. The first two stages belong to the strategic phase of the planning cycle. This phase is concerned with formulating medium-to-long-term objectives, say over a 5-10 year time horizon. The remaining stages are referred to as tactical (stage 3) and operational (stages 4 and 5) and have correspondingly shorter time-frames. It is felt that stages 1 and 2 are presently most relevant; the remaining stages becoming material only after the formal (or informal) introduction of a nutrition policy.

Briefly, what is meant by 'problem recognition' and 'problem formulation'?

Stage 1: problem recognition. Historical information showing the trends in target diseases, plus data on changes in national eating habits, will provide a basis for deciding if a problem of public health dimensions now exists or may be developing. These findings will also indicate the relative seriousness of the problem. By summarizing current knowledge of national dietary and disease patterns and by offering comparisons with neighbouring countries, a surveillance system may contribute substantially to the debate on the need for action and the nature of that action - indeed, it may initiate it.

Stage 2: problem formulation. If it is acknowledged (and not simply by the nutrition community) that a problem does exist, then a detailed formulation of the problem will be needed. Questions, such as the following become germane: Who is improperly nourished? In what ways? Why? How are conditions changing with time?

Answers to these questions will assist clarification, and focus attention on aspects of the problem which are amenable to action. (Socio-political factors are very likely to play a major part, directly or indirectly, in defining the focus.) At this level, however, only partial answers may be expected from the routine data sets which are utilized for stage 1. Core data (discussed later) must, therefore, necessarily be supplemented by detailed, periodic (probably small-scale) surveys of the general population or selected target groups, or both.

A data base for NS. Before outlining a core data base for NS, it is appropriate to consider the function of national surveys in the planning context. Traditionally, large-scale, government sponsored, nationally representative surveys have been the most apparent choice for obtaining information on population food consumption patterns and nutritional status. These continue to be used on an ad hoc basis in many countries when resources (both financial and trained manpower) become available, which may be very 
infrequent indeed. In such circumstances this approach alone, i.e. occasional national surveys, would fail to provide timely information on a regular basis as is essential for monitoring and forecasting purposes. In addition, national surveys constitute a costly means to generate the basic information required for strategic planning. If, however, adequate resources are available, then it may be argued that national surveys provide valuable baseline data which cannot be obtained by other means. But the concern as to whether this use of resources for collecting data to address initial planning needs is the most effective, and this depends on how clearly we can specify our information requirements, must remain.

In order to provide a specification for potential data sources we first require a conceptual model which reflects the natural food-chain of production, consumption and utilization. To generate this model we reason thus:

nutrient intake, coupled with general health status, determines the nutritional status of the individual;

diet, hence nutrient intake, depends on food consumption patterns both inside and (increasingly) outside the home;

food purchased and consumed in the home depends on: family size, family composition, income and prices, plus cultural and traditional influences;

the availability of food in terms of its quantity, type and quality, is a function of production, imports-exports, and agricultural or industrial utilization, or both.

Core data for NS (indicated in Fig. 2) are derived from statistics on national food production-supply, household surveys, food prices (collectively referred to as 'dietary surveillance'; DS), and morbidity and mortality rates, data on infant feeding practice and birth weight, and height and weight measurements for schoolchildren and adults ('health impact surveillance', see De Wals, 1987; Ferro-Luzzi \& D'Amicis, 1987). These sources are routinely tapped and the data base updated.

DS has, for entirely pragmatic reasons, received a higher priority in Ireland. This is partly due to limited resources, but more importantly, because it provides information that can potentially be acted upon. Decision-makers understandably show a greater interest in information which permits them to respond in some positive fashion, e.g. to produce guidelines, to promote change, or even to legislate (Gormley et al. 1987).

DS functions to collate and assess data pertaining to the national diet in terms of structure (the relative importance of the major food components such as cereals, meat, vegetables, etc., in the national diet); quality (the availability of macro-nutrients, e.g. energy, protein, fat, and dietary fibre); and evolution (the changing dietary profile of the nation with time). For population subgroups we are similarly concerned with structure (the choice of foodstuffs, i.e. family purchasing/consumption patterns); quality (the mean group consumption of macro- and micro-nutrients), and evolution (changes in the pattern of group consumption with time). Data relating to both levels may be derived with more or less confidence from food balance sheets (Food and Agriculture Organization, 1984; Organization for Economic Co-operation and Development, 1985) and household budget surveys. Micro-surveys may be used to glean additional detail from targeted subgroups.

Data quality. Data quality is a general issue in NS, especially in relation to the (mis-)use of food balance sheets. There is a potential misconception between data quality and its relation to decision-making. Davis \& Olson (1984) define quality in terms of fitness which they stress is entirely dependent on the context. Fitness encompasses 


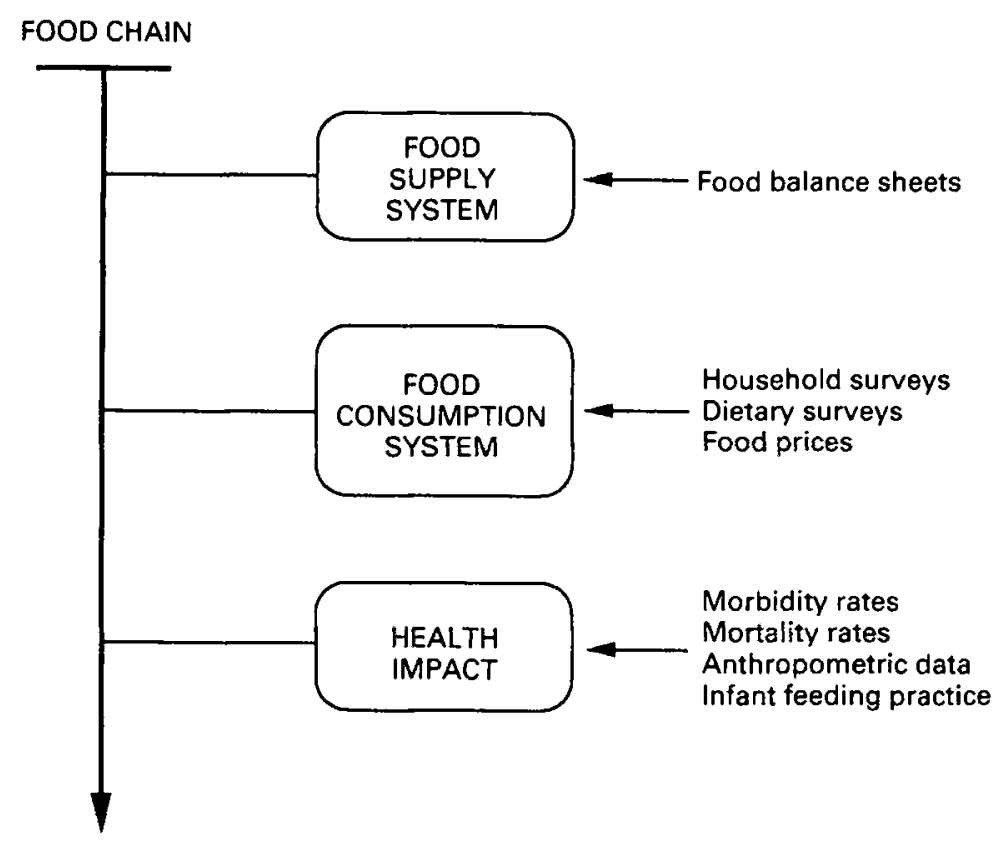

Fig. 2. Core data for a nutritional surveillance system.

such considerations as relevance, precision, accuracy, timeliness, and presentation of results. While it is incumbent upon all concerned in the interpretation and use of surveillance output to effect improvements in the data base, it is also their collective responsibility to appreciate when data of lesser quality will serve.

Relevance. For highest efficiency and effectiveness, information output is designed specifically to cater for the needs of the user. When the role of the system is primarily motivational, then neither efficiency nor effectiveness can be expected to be optimized. This recommends an initially liberal approach to the scope and content of an NS data base with special attention to trend data.

Precision. Härö (1980) reminds us that a high degree of precision is rarely crucial to the decision-making process, and certainly not for strategic planning. Strategic decisions are usually insensitive to precision, order of magnitude estimates suffice. Precision is not primarily at issue for those data sets which are effectively censuses, e.g. the food balance sheet, and national morbidity and mortality rates. It is important in the analysis of surveys and longitudinal studies, e.g. the household budget survey, or nutrition surveys.

Accuracy. We require only that degree of accuracy necessary for the user to reach a decision. Inaccurate or biased estimates of a variable may result from a faulty definition, incomplete data, or poor measurement technique. Any data set, e.g. the food balance sheet, must be considered with each of these in mind, and estimates of the size and direction of errors (often quantifiable by the compiler, i.e. the Central Statistics Office) will be of considerable help in any interpretation. Tukey (1979) warns against the temptation to measure the wrong thing, simply because it can be measured well; what statisticians call, with tongue firmly in cheek, a type III error.

Timeliness. Information which is completely up to date is crucial to success in the 
world of business. For strategic planning, this dependence is less acute. Measurement of the present level of a variable is usually of less interest than establishing how that variable is changing with time.

Output. This relates to the concept of information value, and requires that findings be clearly presented in a form that is both concise and useful to the policy-maker. Appreciating what is intended by 'useful' may again be a problem without suitable feedback from the planner. Clarity will be enhanced by judicious use of graphics and tables (Mahon, 1977). Three separate purposes may be distinguished, i.e. to stimulate, to persuade, or to inform, and each of these, in turn or collectively, must be seen as a legitimate function of the surveillance programme.

Finally, it is worth recalling Boruch's (1984) astute observation that data quality has no clear empirical, conceptual or theoretical linkage to the actual uses to which data are put.

\section{PRACTICE}

Analysis. The analyst is faced with a large volume of dictary (or other) data for which a synthesis must be provided. As has been noted previously, effective communication of information is critical (Clark, 1983). There are two elements essential to this: proper data analysis and good presentation of key results. A detailed exposition will not be possible here but a number of practical pointers may be offered (discussed at length in Kelly, 1989b).

Presentation. In studying or presenting a time series, it is recommended that some form of smoothing be applied. This will remove the typical annual spikes (a fcature of most food series) which might otherwise obscure the long-term data patterns. Three general smoothing techniques which perform well are combined running medians, spline smoothers, and robust locally weighted regression. While the first of these is essentially automatic and quick to apply, the remaining two are more sophisticated and require experience. A variation on the spline smoother, the penalized least squares technique (see Silverman, 1985), has been found to be the most flexible, enabling the calculation of confidence intervals for the smoothed curve.

A proper understanding of the structure and evolution of the nation's diet implies the need to work in many dimensions. Similarly, cross-national comparisons will benefit from a multivariate perspective. A number of data-analytic techniques are available to reduce and summarize high-dimensional data matrices (say foods-nutrients by time or by country) so that the results may be represented and studied in two- or three-dimensional form. Apart from being an end in itself, such an analysis may suggest a basis for a parametric model. The principal method advocated is correspondence analysis (see Greenacre, 1984) which enables a simultaneous presentation of column profiles (e.g. foods-nutrients) and row profiles (e.g. time-countries) and their inter-relationship.

Forecasting. Formal analyses have a role to play in NS in at least two areas: (1) in attempting to understand the relationships within and between food series, plus such exogenous factors as population structure, (2) for forecasting future trends. While obviously related, of these two purposes the latter is of greater practical significance. Forecasts are required by planners to direct policy choices. In order to prepare forecasts, we first require a model of the series of interest. The simplest, and commonest method is based on simple trend analysis. This approach cannot in general be recommended without proper precautions against model mis-specification (especially autocorrelated 
error structure). Poor modelling will seriously bias any forecasts and the end result will be a loss of credibility.

Multiple-regression models provide a natural extension to trend analysis. By including variables other than, or in addition to, time in the model, we could reasonably expect an improvement in forecast performance. A limitation of multiple regression for forecasting is the requirement for future values of the independent variables in order to predict the dependent variable of interest (e.g. \% fat-energy). Discounted least squares (DLS, also known as double exponential smoothing), being a projection method, does not suffer this difficulty. DLS has been found to be a feasible and easily implemented method for obtaining accurate short-term forecasts. The rationale and modelling details are provided by Chatfield (1978) and Abraham \& Ledolter (1983).

Interpretation. In Ireland, the Nutritional Surveillance Unit has responsibility for the information system: system design, data collection, data analysis, and presentation of findings. No interpretation, as such, of the observed trends in diet or disease patterns is offered; presentation is confined to describing these patterns. The importance of offering a careful interpretation of findings for use by policy-makers had been recognized at an early stage. Thus, nominees from various government agencies and national bodies having an interest in food, nutrition, health, education, and official statistics, were invited by the Minister for Health to participate in the programme. The committee, designated the Technical Interpretation Committee (TIC), met for the first time in 1986. The TIC's function has been to advise in relation to three broad areas:

1. to assist in improving data inputs to the surveillance system,

2. to offer guidance on prioritizing work.

3. to offer a critical appraisal of the programme and its findings.

The TIC advises on methodology, and the content and presentation of the findings. Having set out the available information, what interpretation is possible? Following an agreed interpretation, what are the implications? The TIC may offer a set of policy options to the Department of Health with some indication of priorities, and set out a number of recommendations for action.

\section{CONCLUSIONS}

Nutrition planning in a multi-sectoral context presents formidable political and administrative problems; its goal is the control of nutrition disorders and the promotion of lifestyle patterns conducive to healthy behaviour in the population. This demands of any government a considerable investment of time, effort, finance, and fundamentally, the political will to formulate and actively pursue a comprehensive and integrated nutrition policy. Information is a prerequisite for efficient and effective policy-making; an information support system must provide for problem definition, identify the conditioning factors against which strategies and programmes must be planned, and evaluate progress by continuously monitoring all relevant data required to maintain an awareness of the nutritional status of the population. NS is a system's approach designed to meet these needs, whether a nutrition policy is presently in place, or is being considered.

So much for the potential, what of present reality? The capacity of an NS system to actually benefit policy-making depends not only on its ability to 'deliver the goods', but, and this is crucial, on the willingness of policy-makers to use the system to formulate and 
answer basic questions. To date, it must be acknowledged, the department of Health has been a relatively passive recipient of information, rather than a dynamic partner driving the surveillance programme. It is vital that NS as presently implemented (i.e. type 4, maintaining a watching brief) be allowed to mature into that form required for active support to policy development and implementation (type 3). Otherwise, there is a clear danger that the exercise becomes merely academic with minimal utility, especially to planning. The necessary steps may be readily specified:

1. a re-appraisal of the function and present responsibilities of the NS programme by the Department of Health,

2. realistic funding to carry through those responsibilities,

3. a statutory body with a formal mandate to oversee, evaluate and advise the Department on nutrition policy matters.

In retrospect, the last 10 years has seen considerable progress in the development of NS and related activities in Ireland. However, as we enter a second decade one must be concerned that, as yet, only the shadow and not the substance of the recommendations of the Food Advisory Committee (1979) have been implemented. Let us not hasten too slowly!

\section{REFERENCES}

Abraham, B. \& Ledolter, J. (1983). Statistical Methods for Forecasting. New York: Wiley \& Sons.

ACC/SCN (1989). Suggested Approaches for Nutritional Surveillance with Particular Reference to Structural Adjustment. Working Paper, Administrative Committee on Coordination/Sub-Committee on Nutrition. Geneva: WHO.

Boruch, R. (1984). Research on the use of statistical data. In Proceedings of the Social Statistics Section of the American Statistical Association, 1984. Washington, DC: American Statistical Association.

Chatfield, C. (1978). The Holt-Winters forecasting procedure. Journal of the Royal Statistical Society 27C, 264-279.

Clark, N. (1983). Statistical presentation - of what, to whom, and for which purpose? In Proceedings of the Social Statistics Section of the American Statistical Association, 1983. Washington, DC: American Statistical Association.

Davis, G. \& Olson, M. (1984). Management Information Systems: Concepttal Foundations, Structure, and Development. New York: McGraw-Hill.

De Wals, P. (1987). Use of Mortality and Morbidity Statistics for Nutritional Surveillance. In Nutritional Surveillance in Europe: A Critical Appraisal. Annex II - EURONUT Report no. 9, pp. 116-127 [A. Kelly, editor]. Wageningen, The Netherlands: Agricultural University, Wageningen.

Ferro-Luzzi, A. \& D'Amicis. A. (1987). Scope for Nutritional Anthropometry within a Nutritional Surveillance System in Europe. In Nutritional Surveillance in Europe: A Critical Appraisal. Annex III EURONUT Report no. 9, pp. 128-140 [A. Kelly, editor]. Wageningen, The Netherlands: Agricultural University, Wageningen.

Food Advisory Committee (IRE) (1979). Considerations for a Food and Nutrition Policy in Ireland. Dublin: Department of Health.

Food and Agriculture Organization (1984). Food Balance Sheets, 1979-81 Average. Rome: FAO.

Gormley, T., Downey, G. \& O'Beime, D. (1987). Food, Health and the Consumer. Brussels: FAST, CEC.

Greenacre, M. (1984). Theory and Applications of Correspondence Analysis. London: Academic Press.

Habicht, J.-P. (1988). Nutritional surveillance. Working paper for the US AID Workshop on Nutritional Surveillance, Annapolis. Washington, DC: United States Agency for International Development.

Härö, A. (1980). Information systems for health services at the national level. In Information Systems for the Health Services. Public Health in Europe no. 13. Copenhagen: WHO/EURO.

Kelly, A. (1986). Nutritional Surveillance in Ireland: Report for 1985. Dublin: Medico-Social Research Board.

Kelly, A. (editor) (1987). Nutritional Surveillance in Europe: A Critical Appraisal. EURONUT Report no. 9. Wageningen, The Netherlands: Agricultural University, Wageningen. 
Kelly, A. (1989a). Nutritional Surveillance in Ireland: Part I: The National Diet 1961-1986 with Forecasts to 1992. Dublin: Department of Health, Ireland.

Kelly, A. (1989b). Rationale and Methodology for Nutritional Surveillance in Ireland: A Report to the Irish Department of Health. Dublin: Department of Health, Ireland.

Kelly, A. \& Kevany. J. (1985). Nutritional Surveillance in Ireland: Report for 1984. Dublin: Medico-Social Research Board.

Mahon, B. H. (1977). Statistics and Decisions: The Importance of Communication and the Power of Graphical Presentation. Journal of the Royal Statistical Society 140A, 298-307.

Mason, J. (1987). Report of the ACC/SCN Working Group on Nutritional Surveillance: Proposal for Expanding Nutritional Surveillance. SCN 87/NSURVL, Administrative Committee on Coordination/Sub-Committce on Nutrition. Geneva: WHO.

Mason, J., Habicht, J.-P., Tabatabai, H. \& Valverde, V. (1984). Nutritional Surveillance. Geneva: WHO.

Organization for Economic Co-operation and Development (1985). Food Consumption Statistics, $1973-1982$. Paris: OECD.

Rothe, G. E. \& Habicht, J.-P. (1990). Nutritional surveillance: State of the art. In World Food Issues, [M. C. Latham and T. Brun, editors]. New York: Center for the Analysis of World Food Issues, Cornell University (In the Press).

Silverman, B. W. (1985). Some aspects of the spline smoothing approach to non-parametric regression curve fitting. Journal of the Royal Statistical Society 47B, 1-52.

Tukey, J. (1979). Methodology, and the statistician's responsibility for both accuracy and relcvance. Journal of the American Statistical Association 74, 786-793.

World Health Organization (1976). Methodology of nutritional surveillance. Technical Report Series no. 593. Geneva: WHO.

WHO/EURO (1990). Food and Health in Europe: Information Systems for Nutrition Policy Making. Copenhagen: Nutrition Unit, WHO/EURO (In the Press). 